Reprod. Nutr. Dévelop., 1986, 26 (1 A), 85-95.

\title{
Stimulation physico-chimique d'origine alimentaire et motricité digestive chez le lapin
}

\author{
M. PAIRET, Th. BOUYSSOU, A. AUVERGNE (*), \\ M. CANDAU $(*)$, Y. RUCKEBUSCH
}

Laboratoire de Physiologie, Ecole Nationale Vétérinaire, 23, chemin des Capelles, 31076 Toulouse Cedex, France.

(*) Service de Zootechnie et des Productions Animales, Ecole Nationale Supérieure Agronomique,

145, avenue du Muret, 31076 Toulouse Cedex, France.

Summary. Physical-chemical stimulation by the diet and gastrointestinal motility in the rabbit.

The effects on gastrointestinal motility of two different diets, one containing dehydrated lucerne and the other dehydrated beet pulp (both being either coarsely or finely ground before pelleting) were studied in 16 unanesthetized 50-60-day old rabbits fed ad libitum. The rabbits fed with lucerne had better antroduodenal and ileo-caecal coordination, a higher level of electrical activity on the duodenum, and more frequent migrating myoelectric complexes on the jejunum and ileum than those fed with beet pulp. Furthermore, the rabbits fed the finely ground pellets showed weak electrical activity on the ileum and poor ileo-caecal coordination irrespective of fiber source, suggesting a unique effect of size per se on these portions of the digestive tract.

\section{Introduction.}

L'influence sur le transit digestif de la structure physique d'un aliment, en particulier la finesse de mouture avant granulation, peut être aussi importante chez le lapin (Laplace et Lebas, 1977) que celle liée aux produits issus de leur fermentation dans le segment caeco-colique (Fioramonti et al., 1978). En première approximation, on admet que la présence de particules grossières favorise le transit, alors que les acides gras volatils exercent plutôt un effet opposé (Svendsen, 1972).

Comme le transit digestif est lui-même fonction, moins des variations globales de la motricité, que de celles plus spécifiques de la zone antro-duodénale pour la vitesse de l'évacuation gastrique, de la jonction iléo-cæacale (Fioramonti et Ruckebusch, 1974 ; Ehrlein et Ruoff, 1982) et du côlon (Ruckebusch et Hörnicke, 1977) pour la formation de deux types de fèces, nous envisagerons dans cette étude : (i) les particularités motrices gastro-duodénale et iléo-cæcale résultant d'une part de la mouture et d'autre part de la nature des constituants membranaires et (ii) cela pour deux régimes : I'un à base de luzerne, l'autre plus fermentescible à base de pulpe de betterave (Candau et al., 1978a). 


\section{Matériel et méthodes.}

1. - Les aliments. - Deux aliments complets, l'un à base de luzerne déshydratée, l'autre à base de pulpe de betterave déshydratée (tabl. 1) sont présentés sous forme de granulés $(5 \mathrm{~mm}$ de diamètre) à des lapereaux à partir du sevrage ( 30 jours). Pour chaque aliment, deux finesses de mouture, obtenues avant granulation, sont expérimentées et caractérisées par le diamètre de perforation de la grille : $1 \mathrm{~mm}$ ou $4 \mathrm{~mm}$ (tabl. 2). Les aliments seront dénommés par la suite luzerne $1 \mathrm{~mm}\left(\mathrm{~L}_{1}\right)$ ou $4 \mathrm{~mm}\left(\mathrm{~L}_{4}\right)$ et pulpe $1 \mathrm{~mm}\left(\mathrm{P}_{1}\right)$ ou $4 \mathrm{~mm}\left(\mathrm{P}_{4}\right)$.

\section{TABLEAU 1}

Composition centésimale (exprimée en \% d'aliment) et composition analytique (exprimée en \% de la matière sèche) des aliments expérimentaux.

\begin{tabular}{|c|c|c|c|c|}
\hline Régime & \multicolumn{2}{|c|}{ Luzerne } & \multicolumn{2}{|c|}{ Pulpe } \\
\hline Avoine & \multicolumn{2}{|c|}{10} & \multicolumn{2}{|c|}{10} \\
\hline Tourteau de soja 50 & \multicolumn{2}{|c|}{5} & \multicolumn{2}{|c|}{12} \\
\hline Tourteau de tournesol 37 & \multicolumn{2}{|c|}{10} & \multicolumn{2}{|c|}{10} \\
\hline Orge & \multirow{2}{*}{\multicolumn{2}{|c|}{30}} & \multicolumn{2}{|c|}{13} \\
\hline Luzerne déshydratée & \multirow{2}{*}{\multicolumn{2}{|c|}{-}} & & \\
\hline Pulpe de betterave déshydratée & & & \multicolumn{2}{|c|}{50} \\
\hline \multirow[t]{2}{*}{ C.M.V. } & \multicolumn{2}{|c|}{5} & \multicolumn{2}{|c|}{5} \\
\hline & $1 \mathrm{~mm}$ & $4 \mathrm{~mm}$ & $1 \mathrm{~mm}$ & $4 \mathrm{~mm}$ \\
\hline Matière sèche & 89,5 & 88,2 & 90,8 & 90,1 \\
\hline E.N.A. & 57,9 & 56,4 & 64,3 & 64,1 \\
\hline Matières azotées totales & 16,1 & 16,2 & 16,6 & 16,1 \\
\hline Cellulose brute & 14,9 & 16,3 & 12,3 & 13,1 \\
\hline Matières grasses & 1,9 & 2,0 & 1,4 & 1,4 \\
\hline Matières minérales & 9,2 & 9,1 & 5,4 & 5,3 \\
\hline Lignocellulose (Acid Detergent Fiber) & 18,5 & 20,4 & 17,2 & 17,8 \\
\hline Neutral Detergent Fiber & 30,6 & 34,2 & 37,2 & 38,1 \\
\hline Cellulose vraie & 13,0 & 14,3 & 13,7 & 13,6 \\
\hline Hémicellulose & 12,1 & 13,7 & 20,1 & 20,4 \\
\hline Lignine & 5,5 & 6,1 & 3,4 & 4,3 \\
\hline
\end{tabular}

TABLEAU 2

Profil granulométrique par voie humide (exprimée en \% de la matière sèche).

\begin{tabular}{crrrrrr}
\hline $\begin{array}{c}\text { Taille des particules } \\
(\mathrm{mm})\end{array}$ & $>1$ & $>0,5$ & $>0,315$ & $>0,100$ & $>0,05$ & $\begin{array}{c}\text { Soluble } \\
\text { fine }\end{array}$ \\
\hline$L_{\uparrow}$ & 4,5 & 15,6 & 11,9 & 15,0 & 5,4 & 47,5 \\
$\mathrm{~L}_{4}$ & 13,1 & 13,8 & 9,8 & 14,4 & 5,0 & 43,9 \\
\hline $\mathrm{P}_{1}$ & 11,1 & 20,2 & 12,3 & 13,9 & 5,2 & 37,4 \\
$\mathrm{P}_{4}$ & 16,2 & 18,7 & 11,0 & 12,1 & 3,4 & 36,7 \\
\hline
\end{tabular}


La détermination de la matière sèche est effectuée par passage à l'étuve à $103^{\circ} \mathrm{C}$ pendant $24 \mathrm{~h}$ et pesée à poids constant.

Le fractionnement glucidique est réalisé par les méthodes de Van Soest (1967) (Acid Detergent Fiber, Neutral Detergent Fiber, méthode de dosage de la lignine au permanganate) sur $1 \mathrm{~g}$ de contenu sec.

L'azote total est mesuré par la méthode de Kjeldahl (1939).

Les valeurs d'extractif non azoté (ENA), hémicellulose, cellulose sont obtenues par calcul:
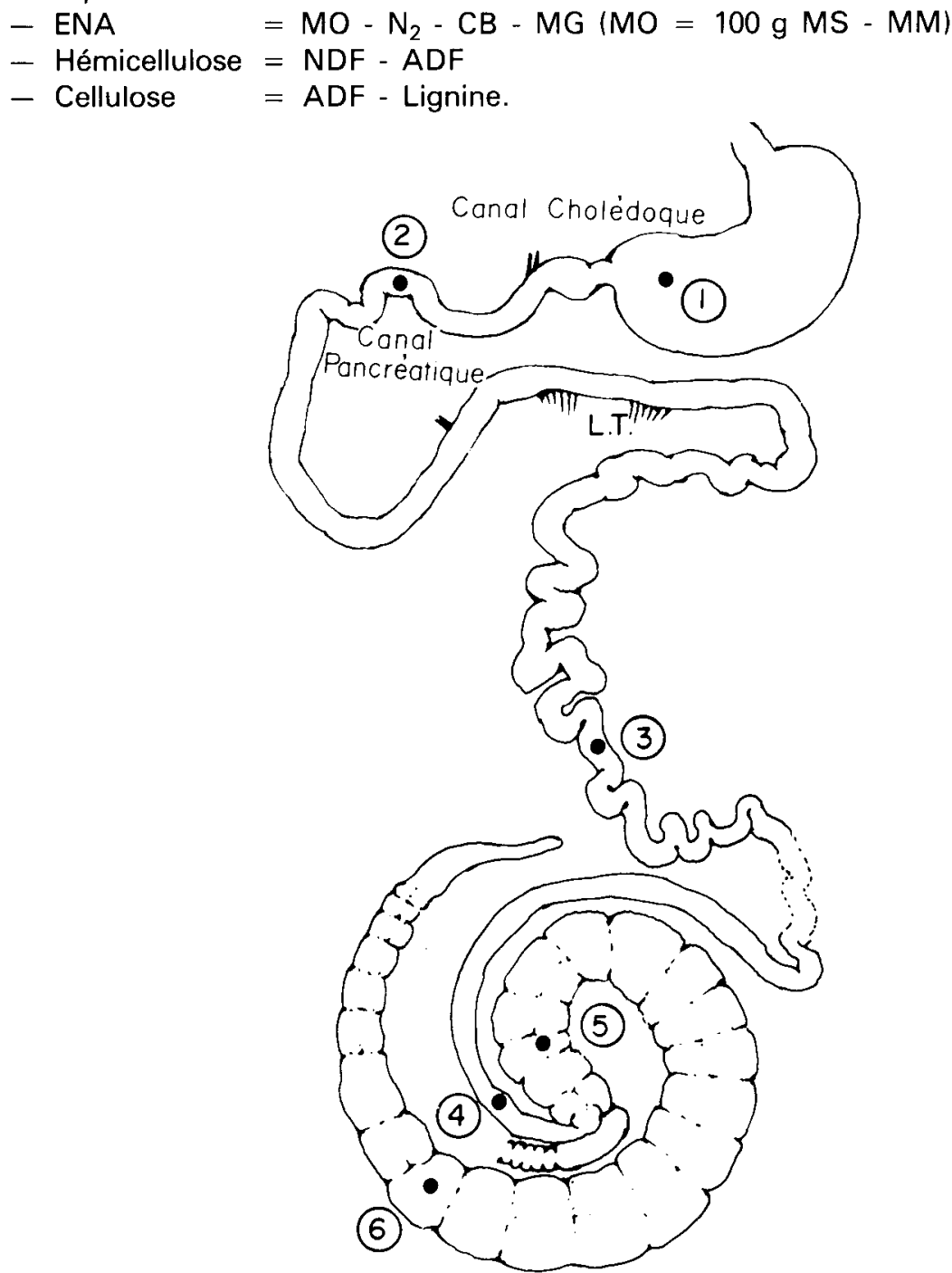

FIG. 1. - Position des électrodes dont les extrémités sont laissées à demeure le long du tractus digestif du lapin. Les groupes $\mathrm{n}^{\circ} 1$ sont fixés dans la paroi antrale à $2 \mathrm{~cm}$ du pylore. La position des autres groupes est pour le duodénum à $20 \mathrm{~cm}$ en aval du pylore (2), pour le jéjunum à $50 \mathrm{~cm}$ après le ligament de Treitz (3) et à $5 \mathrm{~cm}$ de la valvule iléo-cæacale pour l'iléon (4). Les électrodes sont fixées à la hauteur de la $3^{\text {e }}$ spire (5) et de la $17^{\text {e }}$ spire (6) du cæcum. 
Les mesures de granulométrie ont été obtenues par séparation sur des tamis à mailles carrées de $1,0-0,5-0,315-0,100-0,050 \mathrm{~mm}$ de côté.

Mouture : $4 \mathrm{~mm}$ (passage dans un broyeur à marteau avec une grille de $4 \mathrm{~mm}$ ) ; $1 \mathrm{~mm}$ (passage dans un broyeur à marteau avec une grille de $1 \mathrm{~mm}$ ).

Ces quatre lots d'aliment ont ensuite été agglomérés sous la forme de granulés de $5 \mathrm{~mm}$ de diamètre.

La détermination de la granulométrie a été effectuée sur granulés délités durant la nuit (17 h $-8 \mathrm{~h})$, à l'aide d'un appareil "Tamisor " équipé de tamis à mailles carrées.

Le temps de tamisage a été de $10 \mathrm{~min}$ pour chaque échantillon. Chaque plateau a ensuite été placé à l'étuve à $103^{\circ} \mathrm{C}$ pendant $24 \mathrm{~h}$ et pesé de façon à déterminer une répartition du poids de l'échantillon en fonction de la taille des particules.

2. - Motricité digestive. - A l'âge de 56-60 jours, 16 animaux, à jeun depuis $48 \mathrm{~h}$, sont anesthésiés au penthiobarbital sodique $(20 \mathrm{mg} / \mathrm{kg})$; une laparatomie xypho-ombilicale permet de fixer les extrémités dénudées de fils de nickel-chrome émaillé $(120 \mu \mathrm{m}$ de diamètre) dans la paroi digestive (fig. 1). La motricité digestive est appréciée par électromyographie (Ruckebusch, 1973). L'activité électrique recueillie à partir des électrodes est enregistrée sur un électrographe multivoies (Reega VIII, Alvar, Paris), en vue d'évaluer par examen direct le degré de coordination antro-duodénale et iléo-cæacale. L'intégration toutes les $20 \mathrm{~s}$ de l'électromyogramme jéjunal grâce au procédé mis au point par Latour (1973) permet d'identifier la présence de complexes myoélectriques migrants (Ruckebusch et al., 1985).

Les animaux disposent d'eau en permanence et reçoivent leur aliment $\left(L_{1}, P_{1}\right.$, $\left.L_{4}, P_{4}\right)$ à volonté. Les enregistrements directs et intégrés ont été réalisés dès le retour à la consommation ante-opératoire $(7$ à 8 jours).

Les enregistrements durent $6 \mathrm{~h}$, de $9 \mathrm{~h}$ à $15 \mathrm{~h}$. Les lapins sont pris dans leur cage d'élevage, sans jeûne préalable, et placés dans des cages à contention, empêchant prise de nourriture et cæcotrophie. Chaque lapin est soumis à deux séances d'enregistrement.

Les analyses statistiques ont été réalisées à partir du test non paramétrique de Mann et Whitney.

\section{Résultats.}

\section{1. - Composition et profil granulométrique des aliments expérimentaux.}

Les aliments expérimentaux sont isoazotés (tabl. 1) et iso-cellulosiques (cellulose «Van Soest "). Ils diffèrent par contre, quant à leurs teneurs en hémicellulose (plus élevée dans l'aliment pulpe) et en lignine (plus élevée dans l'aliment luzerne).

On note des différences sensibles des teneurs en constituants membranaires en fonction de la finesse de mouture; elles sont, vraisemblablement, dues à une sur- ou sous-estimation lors du dosage suivant la taille des particules. 
L'aliment pulpe, dont la teneur en extractif non azoté (fraction la plus facilement hydrolysable) est supérieure, correspond à l'aliment le plus énergétique.

Pour un même aliment, l'effet granulométrie ne semble jouer que sur les particules de taille supérieure à $1 \mathrm{~mm}$ (mouture $4 \mathrm{~mm}$ ) et celles comprises entre $1 \mathrm{~mm}$ et $0,5 \mathrm{~mm}$ (mouture $1 \mathrm{~mm}$ ) (tabl. 2).

La comparaison du profil granulométrique d'un régime à l'autre paraît plus difficile. En effet, le délitage de l'aliment pulpe favorise le gonflement et l'agrégation des particules, conduisant à une sur-estimation du pourcentage des grosses particules.

\section{2. - Motricité gastro-intestinale.}

a) Motricité antro-duodénale. - L'électromyogramme antral comprend des salves de potentiels, dont la fréquence de répétition varie de 1,5 à 2,3 par minute. La fréquence moyenne la plus élevée des contractions antrales a été obtenue pour I'aliment pulpe, quelle que soit la finesse de mouture (tabl. 3). Les nombreuses salves de potentiels qui surchargent les ondes lentes de l'électromyogramme duodénal constituent l'activité rapide. Celle-ci est significativement plus intense ( $p<0,01$ ) chez les lapins recevant l'aliment luzerne, puisque $50 \%$ des ondes lentes sont surchargées de salves de potentiels, contre $30 \%$ pour les lapins recevant l'aliment pulpe. Aucune différence imputable à la finesse de mouture n'est observée.

TABLEAU 3

Influence du régime et de la finesse de mouture sur la motricité antro-duodénale.

\begin{tabular}{lcccccc}
\hline Régime : & \multicolumn{2}{c}{ Luzerne } & \multicolumn{2}{c}{ Pulpe } & \multicolumn{2}{c}{ Ecart moyen entre: } \\
\hline $\begin{array}{l}\text { Mouture } \\
\text { (effectifs) }\end{array}$ & $1 \mathrm{~mm}$ & $4 \mathrm{~mm}$ & $1 \mathrm{~mm}$ & $4 \underset{(4)}{\mathrm{mm}}$ & Régimes & Moutures \\
\hline $\begin{array}{l}\text { Critères : } \\
\begin{array}{l}\text { Nombre moyen } \\
\text { de contractions } \\
\text { antrales/min }\end{array}\end{array}$ & 1,81 & 1,57 & 2,31 & 2,28 & $-0,61^{* *}$ & $\mathrm{NS}$ \\
\hline $\begin{array}{l}\text { Activité rapide } \\
\text { duodénale }(\%)\end{array}$ & 44,34 & 55,08 & 29,34 & 30,11 & $+19,99^{* *}$ & $\mathrm{NS}$ \\
\hline $\begin{array}{l}\text { Coordination } \\
\text { antro-duodénale }\end{array}$ & 85,15 & 81,06 & 55,32 & 52,90 & $+28,99^{*}$ & $\mathrm{NS}$ \\
\hline
\end{tabular}

** : Effet significatif au seuil 0,$01 ;{ }^{*}$ : Effet significatif au seuil 0,$05 ;$ NS : Effet non significatif.

L'activité électrique rapide correspond au pourcentage d'ondes lentes surchargées de potentiels de pointe.

La coordination antro-duodénale est appréciée à partir du pourcentage de salves de potentiels sur I'antre associées à un groupe nettement défini de 2 à 6 potentiels de pointe sur le duodénum.

Enfin, la coordination antro-duodénale est meilleure $(p<0,05)$ dans le cas de l'aliment luzerne, quelle que soit la taille des particules (fig. 2).

b) Motricité jéjuno-iléale. - L'activité électrique intestinale est organisée, sur le jéjunum et l'iléon, en complexes myoélectriques migrants (CMM), dont la fré- 
quence d'apparition est plus élevée $(p<0,01)$ chez les lapins recevant l'aliment luzerne (tabl. 4 et fig. 2), sans différence liée à la taille des particules. En revanche, la fréquence des CMM au niveau iléal est diminuée $(p<0,10)$ dans le cas des particules de faible taille.

L'activité rapide de l'iléon, correspondant au pourcentage d'ondes lentes surchargées de potentiels de pointe, est affectée de façon significative $(p<0,01)$ par la finesse de mouture des aliments.

\section{TABLEAU 4}

Estimations des effets régime et mouture sur la motricité jéjuno-iléale.

\begin{tabular}{|c|c|c|c|c|c|c|}
\hline \multirow{2}{*}{$\begin{array}{l}\text { Régime : } \\
\begin{array}{l}\text { Mouture } \\
\text { (effectifs) }\end{array}\end{array}$} & \multicolumn{2}{|c|}{ Luzerne } & \multicolumn{2}{|c|}{ Pulpe } & \multicolumn{2}{|c|}{ Ecart moyen entre : } \\
\hline & $\underset{(4)}{1 \mathrm{~mm}}$ & $\underset{(4)}{4 \mathrm{~mm}}$ & $\underset{(4)}{1 \mathrm{~mm}}$ & $\underset{(4)}{4 \mathrm{~mm}}$ & Régimes & Moutures \\
\hline $\begin{array}{l}\text { Critères: } \\
\text { Nombre moyen } \\
\text { de } C M M / 6 \text { h (jéjunum) }\end{array}$ & 3,50 & 4,25 & 1,50 & 1,33 & $+2,46^{* *}$ & NS \\
\hline $\begin{array}{l}\text { Nombre moyen } \\
\text { de CMM/6 h (iléon) }\end{array}$ & 3,25 & 4,00 & 1,25 & 1,67 & $+2,17^{* *}$ & $+0.59\left({ }^{10}\right)$ \\
\hline $\begin{array}{l}\text { Activité rapide } \\
\text { iléale }(\%)\end{array}$ & 34,25 & 39,65 & 31,63 & 38,73 & NS & $+6,25^{* *}$ \\
\hline
\end{tabular}

** : Effet significatif au seuil 0,$01 ; *$ : Effet significatif au seuil 0,$05 ;\left({ }^{10}\right)$ : Effet significatif au seuil 0,10 ; NS : Non significatif.

\section{TABLEAU 5}

Influence de la nature des glucides membranaires et de la taille des particules sur la motricité caecale.

\begin{tabular}{|c|c|c|c|c|c|c|}
\hline \multirow{2}{*}{$\begin{array}{l}\text { Régime : } \\
\begin{array}{l}\text { Mouture } \\
\text { (effectifs) }\end{array}\end{array}$} & \multicolumn{2}{|c|}{ Luzerne } & \multicolumn{2}{|c|}{ Pulpe } & \multicolumn{2}{|c|}{ Ecart moyen entre: } \\
\hline & $1 \underset{(2)}{\mathrm{mm}}$ & $\begin{array}{c}4 \mathrm{~mm} \\
(2)\end{array}$ & $\begin{array}{c}1 \mathrm{~mm} \\
(2)\end{array}$ & $\underset{(2)}{4 \mathrm{~mm}}$ & Régime & Mouture \\
\hline
\end{tabular}

Critères :

Nombre moyen

de contractions

cacales/mn

\begin{tabular}{|c|c|c|c|c|c|c|}
\hline (à la base du caecum) & 2,22 & 1,92 & 1,67 & 1,46 & $+0,51^{* *}$ & $-0,26^{*}$ \\
\hline \multicolumn{7}{|l|}{$\begin{array}{l}\text { Coordination } \\
\text { iléo-caecale (\%) }\end{array}$} \\
\hline $\begin{array}{l}\mathrm{A} \\
\mathrm{B}\end{array}$ & $\begin{array}{l}83,2 \\
83,7\end{array}$ & $\begin{array}{l}88,5 \\
88,9\end{array}$ & $\begin{array}{l}51,1 \\
55,2\end{array}$ & $\begin{array}{l}60,7 \\
60,2\end{array}$ & $\begin{array}{l}+30,0^{* *} \\
+27,6^{* *}\end{array}$ & $\begin{array}{l}+7,5^{*} \\
+5,1^{*}\end{array}$ \\
\hline $\begin{array}{l}\text { Contractions } \\
\text { base-pointe }(\%)\end{array}$ & 85,4 & 87,6 & 56,5 & 67,6 & $+24,5^{* *}$ & NS \\
\hline $\begin{array}{l}\text { Contractions } \\
\text { pointe-base }(\%)\end{array}$ & 84,2 & 71,2 & 42,8 & 27,1 & $+42,8^{* *}$ & $\left.-14,4^{(10}\right)$ \\
\hline
\end{tabular}

A : \% de contractions cæcales ayant pour origine la base de l'organe, précédées d'un groupe de salves de potentiels sur l'iléon.

B : \% de salves iléales immédiatement suivies d'une contraction de la base du cacum.

NS : Effet non significatif ; * : Effet significatif au seuil 0,$05 ;{ }^{*}$ : Effet significatif au seuil 0,01 ;

(10): Effet significatif au seuil 0,10 . 
c) Motricité iléo-cæcale. - L'électromyogramme du cæcum présente des salves de potentiels, les ondes lentes seules n'étant pas détectables. Les fréquences moyennes les plus élevées des contractions enregistrées à la base du cæcum ( $3^{\mathrm{e}}$ spire) ont été obtenues pour l'aliment luzerne $(p<0,01)$ et les fines moutures (p $<0,05)$ (tabl. 5).

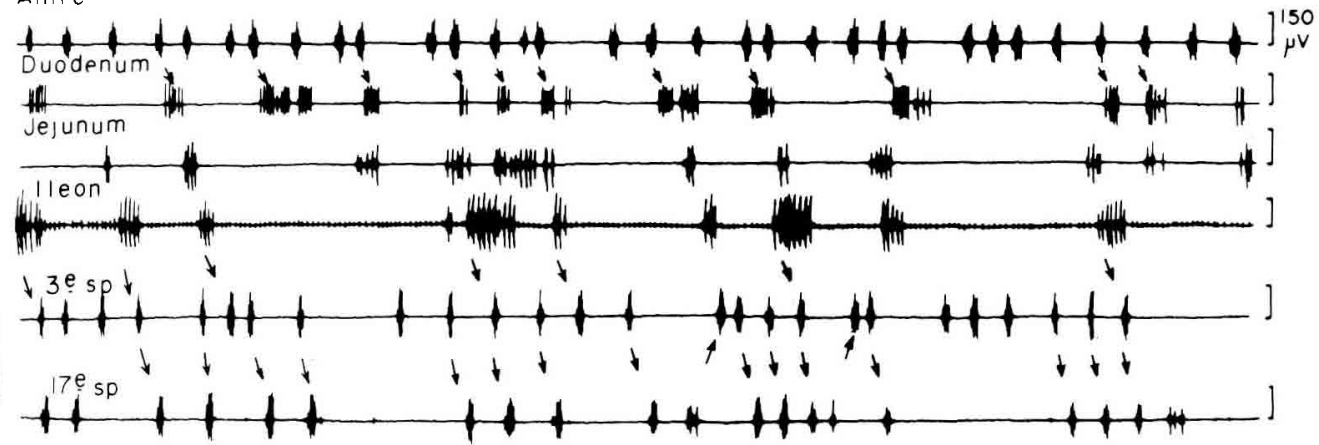

Lapın 1802 L4
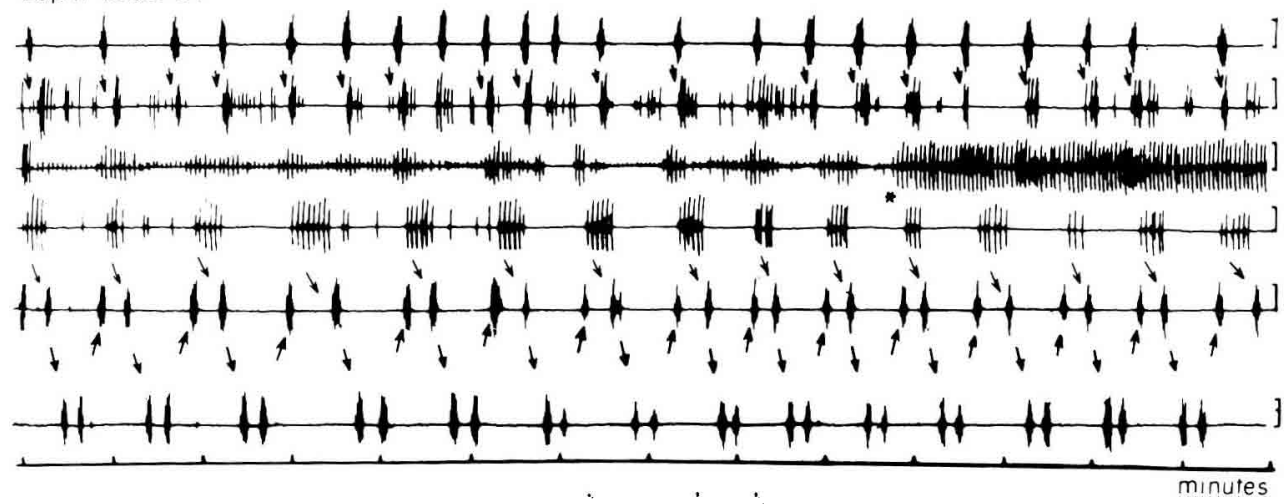

TRACE் INTËGRE

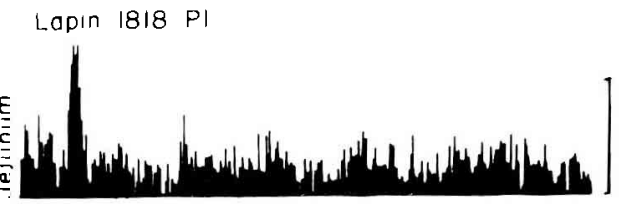

Lanin IBO2 14

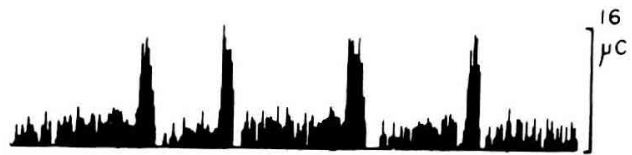

FIG. 2. - Electromyogramme digestif (tracés direct et intégré) de lapins recevant soit l'aliment $P_{1}$, soit l'aliment $L_{4}$. L'aliment $L_{4}$ induit, par rapport à l'aliment $P_{1}:$ - une excellente coordination antro-duodénale $(\backslash)$; - une fréquence d'apparition élevée des complexes myoélectriques (tracé intégré) : - une association régulière de l'activité rapide iléale et des contractions caecales ( \), associée à une propagation de ces contractions de la base vers la pointe $(\backslash)$ de l'organe, puis en sens inverse $(/)$.

* Départ de la phase III d'un complexe myoélectrique sur le jéjunum. 
Quels que soient le régime et la mouture, le rythme des contractions cæcales reste stable au cours des $6 \mathrm{~h}$ d'enregistrement.

Le degré de coordination entre l'iléon et le cæcum a été apprécié en tenant compte du pourcentage des contractions du cæcum qui partent de la base de l'organe en étant précédées d'un groupe défini de 2 à 6 salves de potentiels sur l'iléon et du pourcentage de salves de potentiels enregistrées sur l'iléon et immédiatement suivies d'une contraction de la base du cæacum. Cette coordination entre iléon et cæcum est meilleure pour l'aliment luzerne ( $p<0,01$ ) et les moutures grossières ( $p<0,05$ ), quel que soit le mode d'évaluation (tabl. 5 et fig. 2 ).

Indépendamment de cette caractéristique, une analyse de la propagation des contractions a été effectuée afin d'évaluer l'activité péristaltique (base-pointe) et antipéristaltique (pointe-base) de cet organe.

Les contractions cæcales présentes à la base du cæcum sont classées en deux catégories: (1) les contractions isolées, et les contractions se propageant vers la pointe; (2) les contractions issues de la pointe du cæcum.

Le péristaltisme (base-pointe) est estimé grâce à un pourcentage correspondant au nombre de contractions propagées de la base vers la pointe par rapport à la somme des contractions isolées et propagées (base-pointe). Dans le même sens, pour déterminer l'activité antipéristaltique (pointe-base), deux types de contractions présentes à la pointe du cæcum sont différenciés : (1) contractions isolées et contractions migrant vers la base ; (2) contractions issues de la base.

L'activité antipéristaltique de cet organe (pointe-base) sera donc estimée par le biais d'un pourcentage correspondant au nombre de contractions propagées (pointe-base) par rapport à la somme des contractions isolées et propagées (pointe-base).

Le pourcentage de contractions propagées de la base vers la pointe est supérieur pour l'aliment luzerne $(p<0,01)$. Les contractions propagées dans le sens pointe-base sont favorisées par l'aliment luzerne ( $p<0,01$ ) et les fines moutures $(p<0,10)$ (tabl. 5).

\section{Discussion.}

L'action de l'un des paramètres suivants peut rendre compte des modifications de la motricité digestive induites par les aliments expérimentaux:

- Différences de nature chimique et de structure physique des aliments ; la proportion importante d'hémicellulose vis-à-vis de la lignine rend l'aliment pulpe plus digestible (Candau et al., 1978a).

- Taux de sucres solubles; l'extractif non azoté envisagé sous l'angle de la teneur en énergie de l'aliment fournit, pour le régime pulpe, un aliment à forte teneur en énergie digestible.

- Taille des particules; un broyage fin des constituants d'un aliment avant sa granulation permet d'obtenir une rétention digestive, avec augmentation de la digestibilité apparente (Laplace et Lebas, 1977).

A cela s'ajoute le moment, dans le nycthémère, de la période d'enregistrement. En effet, Prud'hon $(1972,1975)$ a étudié le rythme des prises d'aliment gra- 
nulé sur des groupes de lapins d'âges différents, alimentés ad libitum et totalement libres de leur comportement. Pour des lapins de 8 à 10 semaines, il note une période diurne de faible consommation se situant entre $9 \mathrm{~h}$ et $13 \mathrm{~h}$. L'heure de la plus intense consommation d'aliment se situe entre $20 \mathrm{~h}$ et $1 \mathrm{~h}$. II faut souligner la superposition du rythme des prises d'aliment et du rythme d'excrétion de fèces dures chez des lapins âgés de 8 semaines, établi par Lebas et Laplace (1974).

La cæcotrophie survient pendant la période de consommation faible ou nulle d'aliment associée à la non-excrétion de fèces dures. La durée moyenne est de $7 \mathrm{~h}$, entre $5 \mathrm{~h}$ et $12 \mathrm{~h}$ selon Fioramonti et Ruckebush (1976).

Par conséquent, les enregistrements effectués sur des lapins pris le matin à $9 \mathrm{~h}$ dans leur cage d'élevage, sans jeûne préalable, mais sans possibilité de consommer quoi que ce soit durant l'enregistrement, correspond à la digestion du mélange : aliment-cæcotrophes.

Aussi, les effets rapportés devront tenir compte :

- des caractéristiques de l'aliment (nature des constituants membranaires, concentration en sucres solubles, taille des particules);

- du recyclage des produits issus de la fermentation bactérienne lors de la cæcotrophie (acides gras volatils);

- vraisemblablement, de la teneur en matière sèche du contenu digestif ;

- et enfin, du segment digestif considéré ; par exemple, l'aliment pulpe est responsable d'une hypermotricité antrale quelle que soit la taille des particules.

La faible coordination antroduodénale, associée à une moindre activité duodénale, pourrait être à l'origine, malgré l'hypermotricité antrale, d'une médiocre vidange de l'estomac, entraînant la distension gastrique observée antérieurement (Candau et al., 1978). La présence intraduodénale de substances variées telles que l'acide lactique et l'acide acétique chez le lapin inhibe la vidange gastrique (Mahboubs, 1968) et diminue la motricité du duodénum (Svendsen, 1972). Une telle situation pourrait se concevoir chez les lapins ingérant l'aliment pulpe. En effet, la fermentescibilité supérieure des constituants membranaires de cet aliment rend compte d'une plus grande quantité d'acides gras volatils dans le cacum (Candau et al., 1978b), donc dans les cæecotrophes. La structure des fibres et la teneur en amidon de chaque aliment n'est sûrement pas sans incidence. Une meilleure solubilité de l'aliment pulpe pourrait favoriser son hydrolyse et libérer une plus grande quantité de sucres solubles. La libération d'holosides résultant de la digestion de l'amidon, inhibe la vidange gastrique chez le Porc (Borgida et Laplace, 1977).

La fréquence de répétition des complexes myoélectriques le long du jéjunoiléon (Ruckebusch et al., 1985) est significativement inférieure chez les lapins recevant l'aliment pulpe. Un tel profil moteur chez le porc consommant un aliment standard correspond à une réplétion duodénale importante (Laplace, 1978). Par ailleurs, la perfusion, dans un segment isolé d'intestin grêle de chien, d'une solution de glucides ou de peptides à $7,5 \mathrm{kcal} / \mathrm{kg}$ ou à $15 \mathrm{kcal} / \mathrm{kg}$ déclenche une activité motrice postprandiale dont la durée augmente avec la teneur en calories (Schang et al., 1979).

La question se pose donc de savoir quel est le facteur déterminant la diminution du nombre de complexes chez les lapins ingérant l'aliment pulpe : réplétion 
intestinale importante ou forte teneur en énergie du régime? Une intervention conjointe de ces deux facteurs est également plausible.

Inversement, l'excellente coordination iléo-cæacale et la propagation des contractions cæcales de la base vers la pointe, plus importante avec l'aliment luzerne, permettrait un meilleur remplissage et une meilleure répartition du contenu dans ce réservoir. La propagation pointe-base des contractions, également favorisée par ce même aliment, peut être associée à un meilleur brassage du contenu cæcal.

L'influence de la finesse de mouture de l'aliment sur la motricité digestive apparaît de façon nette sur la partie distale du tube digestif. Au niveau iléal, chez les lapins recevant les fines moutures, on note une activité électrique globale faible et une médiocre coordination iléo-cæcale.

Ce profil moteur favorise-t-il une stase iléale? Ruckebusch et Fioramonti (1975) observent, chez le rat, que lorsque l'injection de bleu de méthylène dans le duodénum a lieu pendant une phase d'activité irrégulière d'un complexe myoélectrique migrant, il y a propulsion du marqueur en direction aborale. Si celui-ci est injecté au cours de la période de quiescence, il reste sur place jusqu'à l'arrivée de la phase d'activité irrégulière du complexe myoélectrique suivant.

Cependant, il est remarquable que l'ingestion de lactose chez le porc (Laplace, 1978) conduise à une augmentation considérable des volumes endoluminaux. Ceux-ci transitent rapidement en présence d'activité électrique faible et disparate. Aussi, afin de vérifier l'exacte relation entre phénomènes électriques de la paroi intestinale et le déplacement des contenus, il serait souhaitable, comme le précise Laplace (1978), d'évaluer les volumes du contenu digestif, la matière sèche et la composition des digesta, en particulier la teneur en acides gras volatils ; ceux-ci inhibent la motricité duodénale chez le lapin (Svendsen, 1972) et celle du côlon chez le chien (Bardon et Fioramonti, 1983). Enfin, il est intéressant de mettre en parallèle, pour les aliments grossiers, une excellente coordination iléo-cæcale et la faible migration des contractions cæcales de la pointe vers la base. Ainsi, une telle situation suggère que pour un aliment de mouture grossière, on observe un remplissage aisé du cæcum, associé à une migration lente vers le côlon proximal.

Cependant, le processus de séparation physique des phases solide et liquide dans le côlon proximal chez le lapin (Björnhag, 1972) au cours de la période de production de fèces dures empêche toute estimation du temps de séjour des aliments dans le cæcum.

En résumé, les différences de motricité digestive observées entre les deux régimes (pulpe et luzerne) relèvent de la nature des constituants membranaires (structure physique et composition chimique) et de la teneur en énergie (quantité de sucres solubles). A cela s'ajoute, en zone iléale et iléo-cæacale, l'influence déterminante de la taille des particules. 
BARDON T., FIORAMONTI J., 1983. Nature of the effects of bran on digestive transit time in pigs. Br. J. Nutr., 50, 685-690.

BJÖRNHAG G., 1972. Separation and delay of contents in the rabbit colon. Swedish J. agric. Res., 2. $125-136$.

BORGIDA L. P., LAPLACE J. P., 1977. Evacuation gastrique comparée de régimes à base de fécule de pomme de terre crue ou extrudée chez le porc en croissance. Ann. Zootech., 26, 585-593.

CANDAU M., BERTRAND B., FIORAMONTI J., 1978a. Variations de la digestibilité des constituants de la ration chez le lapin. C.R. Soc. Biol., 172, 554.

CANDAU M., DELPON G., FIORAMONTI J., 1978b. Influence de la nature des glucides membranaires sur le développement anatomo-fonctionnel du tractus digestif du lapin. 2èmes Journ. Rech. Cunic., Toulouse (communication I).

EHRLEIN H. J., RUOFF G., 1982. Cecal motility and flow of ingesta from the ileum to the cecum, appendix, and colon in rabbits, 157-165. In M. WIENBECK, Motility of the digestive tract, Raven Press, New York.

FIORAMONTI J., RUCKEBUSCH Y., 1974. La motricité cæacale chez le lapin. 1. Nature des contractions. Ann. Rech. vét., 5, 1-13.

FIORAMONTI J., RUCKEBUSCH Y., 1976. La motricité cæcale chez le lapin. 3. Dualité de l'excrétion fécale. Ann. Rech. vét., 7, 281-295.

FIORAMONTI J., BUENO L., CANDAU M., 1978. Motricité digestive et glucides membranaires chez le lapin. 2èmes Journ. Rech. Cunic., Toulouse (communication II).

KJELDALH, 1939, cité par FRIEDRICH et LACOURT. In La pratique de la microanalyse quantitative, Dunod Ed., Paris, 387 p.

LAPLACE J. P., 1978. Observations préliminaires sur quelques effets du lactose chez le porc. Complexes myoélectriques de l'intestin grêle, motricité cæacale et diarrhée. Ann. Zootech., 27, 495-517.

LAPLACE J. P., LEBAS F., 1977. Le transit digestif chez le lapin. VII. Influence de la finesse du broyage des constituants d'un aliment granulé. Ann. Zootech., 26, 413-420.

LATOUR A., 1973. Un dispositif simple d'analyse quantitative de l'électromyogramme intestinal chronique. Ann. Rech. vét., 4, 347-353.

LEBAS F., 1985. Profil granulométrique par voie humide. (Communication personnelle).

LEBAS F., LAPLACE J. P., 1974. Sur l'excrétion fécale chez le lapin. Ann. Zootech., 23, 577-581.

MAHBOURS, 1968. Le cycle entéro-hépatique de l'acide lactique chez le lapin. Th. Doct.-Ing. Paris, $70 \mathrm{p}$.

PRUD'HON M., CARLES Y., GOUSSOPOULOS J., KOEHL P. F., 1972. Enregistrement graphique des concommations d'aliments solide et liquide du lapin domestique nourri ad libitum. Ann. Zootech., 21, 451-460.

PRUD'HON M., CHERUBIN M., GOUSSOPOULOS J., CARLES Y., 1975. Evolution au cours de la croissance, des caractéristiques de la consommation d'aliments solide et liquide du lapin domestique nourri ad libitum. Ann. Zootech., 24, 289-298.

RUCKEBUSCH Y., 1973. L'électromyographie globale des muscles lisses à partir d'électrodes chroniques intrapariétales souples. Revue Méd. vét., 124, 1407-1434.

RUCKEBUSCH M., FIORAMONTI J., 1975. Electrical spiking activity and propulsion in small intestine in fed and fasted rats. Gastroenterology, 68, 1500-1508.

RUCKEBUSCH Y., HÖRNICKE H., 1977. Motility of the rabbit's colon and cecotrophy. Physiol. Behav., 18, 871-878.

RUCKEBUSCH Y., PAIRET M., BECHT J., 1985. Origin and characterization of the migrating myoelectric complex in rabbits. Dig. Dis. Sci., 30, 742-748.

SCHANG, J. C., SAVA P., DAUCHEL J., GRENIER J. F., 1979. Relations entre motricité et absorption intestinales. Etude électromyographique chez le chien. Pathol. Biol., 27, 389-393.

SVENDSEN P., 1972. Inhibition of intestinal motility by volatile fatty acids. In vitro experiments on an isolated rabbit duodenum. Nord. vet. Med., 24, 123-131.

VAN SOEST D. J., 1967. Development of a comprehensive system of feeds analyses and its applications to forage. J. anim. Sci., 26, 119-128. 